\title{
Concomitant THC and stress adolescent exposure induces impaired fear extinction and related neurobiological changes in adulthood
}

Rocio Saravia ${ }^{1 *}$, Marc Ten-Blanco ${ }^{1 * \dagger}$, Marina Julià-Hernández ${ }^{1}$, Humberto Gagliano ${ }^{2,3}$, Raül Andero ${ }^{2,3,4}$, Antonio Armario ${ }^{2,3}$, Rafael Maldonado ${ }^{1 \ddagger}$ and Fernando Berrendero ${ }^{1,5 \ddagger}$

${ }^{1}$ Laboratory of Neuropharmacology, Department of Experimental and Health Sciences, Universitat Pompeu Fabra, PRBB, 08003 Barcelona, Spain

${ }^{2}$ Institute of Neuroscience and Animal Physiology Unit (School of Biosciences), Universitat Autònoma de Barcelona, 08193 Cerdanyola del Vallès, Barcelona, Spain

${ }^{3}$ CIBERSAM, Corporació Sanitaria Parc Taulí, Sabadell, Spain

${ }^{4}$ Department of Psychobiology and Methodology in Health Sciences, Universitat Autònoma de Barcelona, Bellaterra, Spain

${ }^{5}$ Faculty of Experimental Sciences, Universidad Francisco de Vitoria, UFV, 28223 Pozuelo de Alarcón, Madrid, Spain

†Present address: Faculty of Experimental Sciences, Universidad Francisco de Vitoria, UFV, 28223 Pozuelo de Alarcón, Madrid, Spain

*These authors contributed equally to this work

¥Corresponding authors: Rafael Maldonado. Laboratory of Neuropharmacology, Department of Experimental and Health Sciences. Universitat Pompeu Fabra, PRBB, 08003 Barcelona, Spain. Phone: 34-93-316-0824; E-mail: rafael.maldonado@upf.edu. Fernando Berrendero. Faculty of Experimental Sciences, Universidad Francisco de Vitoria, 28223 Pozuelo de Alarcón, Madrid, Spain. Phone: 34-91-709-1400; E-mail: fernando.berrendero@ufv.es 


\section{Abstract}

$\Delta^{9}$-tetrahydrocannabinol (THC) consumption during adolescence is reported to be a risk factor for the appearance of psychiatric disorders later in life. The interaction between genetic or environmental events and cannabinoid exposure in the adolescent period can also contribute to exacerbate behavioural deficits in adulthood. Here we investigate the effects of THC treatment as well as the consequences of concomitant THC and stress exposure during adolescence in the extinction of fear memory in adult mice. Adolescent mice treated with THC and exposed to stress exhibit impaired cued fear extinction in adulthood. However, no effect was observed in animals exposed to these two factors separately. Notably, resistance to fear extinction was associated with decreased neuronal activity in the basolateral amygdala (BLA) and the infralimbic prefrontal cortex, suggesting a long-term dysregulation of the fear circuit. These changes in neuronal activation were paralleled with structural plasticity alterations. Indeed, an increase of immature dendritic spines in pyramidal neurons of the BLA was revealed in mice simultaneously exposed to THC and stress. Corticosterone levels were also enhanced after the cued fear conditioning session in the same experimental group. These results show that an interaction between cannabis exposure and stress during adolescence may lead to long-term anxiety disorders characterized by the presence of pathological fear.

Keywords: $\Delta^{9}$-tetrahydrocannabinol, stress, adolescence, fear extinction, dendritic spines, amygdala 


\section{Introduction}

Cannabis remains the most widely used illicit substance worldwide. The regular use of cannabis often begins during adolescence which is of particular concern because this period is crucial to generate efficient neuronal pathways by constant neuroplastic shaping, synaptic reorganization and neurochemical changes (Sturman and Moghaddam, 2011). Pharmacological and/or environmental factors affecting the endocannabinoid system during the adolescent period can lead to altered brain maturation considering the important role played by this system in the neurodevelopmental changes that occur during this time (Fernández-Ruiz et al, 2000; Harkany et al, 2007). As a consequence, preclinical and epidemiological data suggest that adolescent cannabinoid exposure may increase the risk for the appearance of psychiatric diseases in adult life (Malone et al, 2010; HigueraMatas et al, 2015), including emotional dysregulation. Recent studies relate cannabis use in adolescence with amygdala hypersensitivity to signals of threat (Spechler et al, 2015), and with an increased likelihood of having posttraumatic stress disorder symptoms in adulthood (Lee et al, 2017). Other disturbances associated with adolescent cannabis use may include psychotic-like symptoms, cognitive deficits, and increased addiction vulnerability (Silins et al, 2014; Renard et al, 2016).

Most psychiatric disorders involve multiple ethiopathological factors that can interact across the lifespan and trigger disease onset (Caspi and Moffit, 2006). The interaction between cannabis consumption and genetic or environmental factors during adolescence may have a crucial influence in the detrimental effects of this drug later in life (Rubino and Parolaro, 2016). Adult mice with genetic mutations in some genes involved in schizophrenia (O’Tuathaigh et al, 2012; Long et al, 2013; Ballinger et al, 2015) or exposed to stressful events early in life (Llorente-Berzal et al, 2011; Zamberletti et al, 
2012; Klug and van den Buuse, 2012) showed altered behavioural responses due to $\Delta^{9}$ tetrahydrocannabinol (THC) adolescent treatment. This interaction of cannabis exposure and genetic/environmental events produces protective or negative effects depending on the genetic profile, sex, and stress level (Rubino and Parolaro, 2016).

The most consistent consequences of adolescent cannabinoid exposure are related to long-term impairments in cognitive function. Thus, numerous studies have shown working memory deficits in animals exposed to different cannabinoid agonists during adolescence (Renard et al, 2016). The consequences in emotional memory have been less studied, although adolescent THC administration did not produce lasting effects in this behaviour (Ballinger et al, 2015; Rubino et al, 2009a; Rubino et al, 2009b). However, whether adolescent cannabinoid exposure alone or in combination with environmental factors such as stress affects the extinction of fear memory in adult life remains to be elucidated. Cannabis consumption and stressful events are often associated (Ketcherside and Filbey, 2015). The effects in adulthood of cannabis consumption during adolescence may be exacerbated by stress exposure in this period of life. Moreover, stress and fear responses share common neural circuits, and the neuronal structures involved in fear acquisition and extinction are also highly sensitive to stress effects (Stockhorst and Antov, 2016).

Here, we reveal that concomitant THC and stress adolescent exposure induces long-term impairment in fear extinction. This effect is associated with reduced neuronal activity and structural plasticity changes in key limbic brain regions. These findings suggest that THC chronic consumption under stress conditions during adolescence may increase the risk for the appearance of anxiety disorders related to trauma exposure. 


\section{Materials and Methods}

\subsection{Animals}

Adolescent female and male, and adult male C57BL6/J mice (Charles River, France) were used in these experiments. Animals were housed 3-5 per cage in a room with controlled temperature $\left(21 \pm 1^{\circ} \mathrm{C}\right)$, and humidity $(55 \pm 10 \%)$ and with a $12 \mathrm{~h}$ light/12h dark cycle. All behavioural studies were conducted during the light period. Food and water were available ad libitum. Animal procedures were performed in accordance with the guidelines of the European Communities Council Directive 2010/63/EU and approved by the local ethical committee (CEEA-IMAS-UPF), and the statement of compliance with standards for use of laboratory animals by foreign institutions nr. 5388-01 approved by the National Institutes of Health. All behavioural data were obtained by experimental observers blinded to the experimental conditions.

\subsection{Drugs}

THC stored at $100 \mathrm{mg} / \mathrm{ml}$ in ethanol (THC-Pharm-GmbH, Germany) was diluted in 5\% Tween-80 and physiological saline solution to achieve doses of 3, 6 and $12 \mathrm{mg} / \mathrm{kg}$ (5 $\mathrm{ml} / \mathrm{kg}$ of body weight).

\subsection{Experimental designs}

\subsubsection{Adolescent THC treatment}

The long-term effects of THC administration in anxiety-like responses, locomotor activity, and fear conditioning expression and extinction were evaluated in adolescent 
male and female mice. Adolescence, which is a vulnerable period for the onset of neuropsychiatric disorders, covers the complete time span from childhood (shortly before puberty) to adulthood, including the pubertal period (Schneider, 2013). The timing of human adolescence is difficult to define (Schneider, 2008), and therefore the exact timing of this period in laboratory rodents represents a challenge in animal research (Schneider, 2013). Starting at PND 35, mice were subcutaneously administered with increasing doses of THC (PND 35-39: $3 \mathrm{mg} / \mathrm{kg}$, PND 40-44: $6 \mathrm{mg} / \mathrm{kg}$, and PND 45-49: $12 \mathrm{mg} / \mathrm{kg}$ ) or vehicle during 15 days in order to counter the development of drug tolerance (Renard et al, 2017). Similar protocols using escalating doses of THC during adolescence have been previously used in numerous studies (Rubino et al. 2008; Llorente-Berzal et al. 2013; Cadoni et al. 2015). Moreover, comparable time frames for adolescent cannabinoid treatment in both male and female rodents have been previously reported (Biscaia et al, 2003; Rubino et al, 2008; Realini et al, 2011; Bartolato et al, 2014; Zamberletti et al, 2014). Male and female mice could be at different stages of the development at the onset and during the treatment schedule. However, the objective of our study was to evaluate in adult mice the possible effects of THC treatment during the adolescent period, a crucial life stage for the neuronal development. From PND 50 to 69, animals remained undisturbed. At PND 70, behavioural evaluation was carried out in the order described in Fig. 1A for male and in Fig. S1 for female mice. The interval of time between adolescent THC or vehicle treatment and adult behavioural experiments is similar to those used in previous reports (Quinn et al. 2008; Llorente-Berzal et al. 2013). On the other hand, longterm stress of injection effects during adolescence (Keeley et al, 2015; Simone et al, 2018a; Simone et al, 2018b) have been previously reported, which may reflect a greater vulnerability of adolescent animals to repeated physical stress exposure. However, 
control groups were injected with vehicle in our experimental protocols suggesting that the effects observed in mice treated with THC were due to this drug.

\subsubsection{Concomitant THC and stress adolescent exposure}

To assess the interaction between THC and mild stress, adolescent male mice treated with THC or vehicle, as previously described, were exposed to different stressors: forced swimming, tail suspension and restraint. Similar stressors, applied singly or in combination, either acutely or chronically, have been used in previous studies to investigate the role of stress in fear processing (Maren and Holmes, 2016). At the end of each 5-days vehicle or THC exposure, one stressor was applied as shown in Fig. $2 A$. Moreover, two additional stressors were applied at PND 60 (forced swimming) and 65 (tail suspension) (Fig. 2A). Behavioural evaluation was carried out at PND 70 in the order shown in Fig. $2 A$.

\subsubsection{Concomitant THC and stress adult exposure}

To evaluate whether the long-term effects of simultaneous THC and stress exposure were age dependent, a similar experimental design was performed in adult male mice (Fig. S2A). Starting at PND 56, mice were subcutaneously administered with increasing doses of THC (PND 56-60: 3 mg/kg, PND 61-65: 6 mg/kg, and PND 66-70: $12 \mathrm{mg} / \mathrm{kg}$ ) or vehicle during 15 days. At the end of each 5-days THC exposure one stressor was applied as previously described (Fig $2 A$ and Fig. S2A). Two additional stressors were applied at PND 80 (forced swimming) and 85 (tail suspension). Behavioural evaluation was carried out at PND 90 in the order described in Fig. S2A. 


\subsection{Stress procedure}

\subsubsection{Forced Swimming}

Mice were placed in a clear Plexiglas cylinder containing water $\left(20 \pm 1^{\circ} \mathrm{C}\right)$ for $6 \mathrm{~min}$. The depth of the container and the volume of water were enough to prevent the animal touching the bottom, thus forcing mice to swim.

\subsubsection{Tail Suspension}

Animals were suspended by a $15 \mathrm{~cm}$ thin string hanged from a metal rod and stuck with adhesive tape $1 \mathrm{~cm}$ from the tip of the tail. The front paws were sufficiently distant to the ground to avoid mice touching it. Tail suspension was performed during 6 min.

\subsubsection{Restraint}

Stress was induced by immobilizing the animal with a restrainer apparatus. Mice were placed individually inside a $50 \mathrm{ml}$ conical tube with $0.5 \mathrm{~cm}$ air holes for breathing without access to food or water for $30 \mathrm{~min}$.

\subsection{Behavioural experiments}

\subsubsection{Locomotor Activity}

Changes in horizontal activity were assessed by using locomotor activity boxes $(9 \mathrm{x} 20 \mathrm{x}$

$11 \mathrm{~cm}$, Imetronic, France). Mice were placed in locomotor cages with low luminosity. Activity was measured as the total number of horizontal photocell counts during $15 \mathrm{~min}$.

\subsubsection{Elevated Plus Maze}

Elevated plus maze was performed to evaluate anxiety-like responses (Rubino et al, 2008). The maze consisted of four arms $(16$ x $5 \mathrm{~cm})$ extended from a central square $(5 \mathrm{x}$ 
$5 \mathrm{~cm}$ ) shaping a cross. Two opposite arms were delimited by vertical walls (closed arms), whereas the two other opposite arms had unprotected edges (open arms). The apparatus was elevated $30 \mathrm{~cm}$ above the floor and indirectly illuminated from the top (50-60 lux in the open arm). A 5 min trial was conducted by placing each animal in the central square and facing one of the open arms. The performance was recorded with a video camera system located above the maze. Results are expressed as the total entries to the closed and open arms, and the percentage of time spent in the open arms with respect to the total amount of time spent in both arms. An arm entry was counted when the animal moved both front paws into the arm.

\subsubsection{Fear Conditioning}

Training and testing were conducted as described previously with some modifications (Bilkei-Gorzo et al., 2012; Na et al., 2012; Flores et al., 2014; Soria-Gómez et al., 2015). Mice were individually placed in a shuttle chamber (LE918, Panlab, Barcelona) surrounded by a sound-attenuating cabinet (Flores et al, 2014). The chamber floor was formed by parallel stainless-steel bars connected to a scrambled shock generator. On the training day, mice were habituated to the chamber during $180 \mathrm{~s}$ before the exposure to an acute beeping $30 \mathrm{~s}$ sound $(80 \mathrm{~dB})$ repeated 3 times with a $10 \mathrm{~s}$ silenced interval. Each animal received an unconditioned stimulus (US) (0.7 mA footshock during 2s) paired with the end of each sound (conditioned stimulus, CS). After the third shock, the animal remained $30 \mathrm{~s}$ in the shuttle chamber. To test context-induced fear conditioning, mice were placed in the shuttle chamber $24 \mathrm{~h}$ after the training. Fear memory was assessed as the percentage of time that mice spent freezing during the first 3 min. Freezing response, a rodent's natural response to fear, was evaluated by direct observation and defined as complete lack of movement, except for respiration for more than $1 \mathrm{~s}$. To evaluate cued 
fear conditioning (session 1), mice were re-exposed to the CS in a novel environment (a wide dark cylinder) $24 \mathrm{~h}$ after context acquisition. Mice were allowed to adapt for $3 \mathrm{~min}$ to the new environment which was followed by $30 \mathrm{~s}$ of the sound used in the training day. This sound was repeated 4 times with a $10 \mathrm{~s}$ interval. Freezing was scored during the time the sound was active. After the last sound trial, mice remained in the cylinder for $30 \mathrm{~s}$.

\subsubsection{Extinction training}

Extinction training was initiated $24 \mathrm{~h}$ after the cue-dependent fear conditioning test. Mice were placed in the fear conditioning cylinder with a novel environment as described above. Mice were given once daily extinction training sessions for 5 days (sessions 2-6). The percentage of freezing time was calculated by following the same experimental procedure as in the session 1 . The habituation time was reduced to $1 \mathrm{~min}$ as mice were previously adapted to the new context. Data from fear extinction in adult male mice exposed to THC and stress during the adolescent period were expressed as percentage of freezing behaviour and as area under the curve (AUC). AUC was calculated by using a standard trapezoid method, AUC $=[0.5 \times(B 1+B 2) \times h]+[0.5 \times(B 2+B 3) \times h]+\ldots[0.5$ $\times(\mathrm{Bn}+\mathrm{Bn}+1) \times \mathrm{h}$ ], where $\mathrm{Bn}$ were the percentage of freezing behaviour for each mouse and h was the time (days) passed between the consecutive measurements (Gibaldi and Perrier, 1975).

\subsection{Immunoblot analysis}

Amygdala tissue was extracted 25 days after the last THC or vehicle administration (PND74). Tissue was immediately frozen and store at $-80^{\circ} \mathrm{C}$. Amygdala was homogenized in 30 volumes lysis buffer containing protease and phosphatase inhibitors. Samples were then centrifugated to eliminate any solid residue. Equal amounts of protein 
samples $(20 \mu \mathrm{g} /$ well) were separated in $10 \%$ polyacrylamide gels before electrophoretic transfer onto to nitrocellulose membranes (Bio-Rad). Membranes were blocked during $1 \mathrm{~h}$ in $5 \%$ bovine serum albumin (BSA)-T-TBS prior incubation for $2 \mathrm{~h}$ with the primary antibodies: CB1 cannabinoid receptor (CB1R) (rabbit polyclonal, 1:500) and antiglyceraldehyde-3-phosphate dehydrogenase (GAPDH, mouse monoclonal, 1:15000) form Frontier Institute (Cb1-Rb-Af380-1) and Santa Cruz Biotechnology (sc-32233), respectively. Then, membranes were rinsed 3 times and incubated for $1 \mathrm{~h}$ with their corresponding secondary antibodies coupled to horseradish peroxidase: rabbit (1:10000) and mouse (1:10000) from Cell Signaling. Immunochemiluminescence was produced by incubation of the membranes with West-femto ECL substrate (Thermo Fisher Scientific). Images of immunoreactive bands were acquire on a ChemiDoc XRS System (Bio-Rad) and quantified by The Quantity One software v4.6.3 (Bio-Rad). The values obtained for CB1R were normalized to the detection of GAPDH in the same sample and expressed as a percentage of the control group (Vehicle-Non stress) (Fig. S3).

\subsection{Plasma corticosterone quantification}

Blood samples were collected at different time points: immediately after restraint (PND 49), at PND 67 (basal), and 30 min after training (PND 72), cued-fear conditioning (session 1) (PND 74) and cued-fear extinction (session 5) (PND 78) (Fig. 3A). Blood samples were obtained from the tail in tubes containing ethylenediaminetetraacetic acid. Double-antibody radioimmunoassay (RIA) was used to determine plasma corticosterone levels. RIA used 125I-corticosterone-carboximethyloxime-tyrosine-methyl ester (ICNBiolink 2000, Spain), synthetic corticosterone (Sigma, Spain) as the standard, and an antibody raised in rabbits against corticosteronecarboxi-methyloxime-BSA kindly provided by Dr G. Makara (Institute of Experimental Medicine, Budapest, Hungary). 
Plasma corticosteroid-binding globulin was inactivated by low $\mathrm{pH}$. All samples to be statistically compared were quantified in the same assay to avoid inter-assay variability.

\subsection{Immunofluorescence}

Two h after the last cued fear extinction session or just before behavioural testing, mice were deeply anesthetized by ip injection $(0.2 \mathrm{ml} / 10 \mathrm{~g}$ body weight $)$ of a mixture of ketamine (100 mg/kg) and xylazine (20 mg/kg) prior to rapid intracardiac perfusion. Mice were intracardically perfused with 4\% paraformaldehyde (PFA) solution. Brains were removed from skull and post-fixed in PFA for $24 \mathrm{~h}$ at $4^{\circ} \mathrm{C}$. Then, brains were transferred to a solution of $30 \%$ sucrose in PB $0.1 \mathrm{M}$ and kept at $4^{\circ} \mathrm{C}$. Coronal sections of $30 \mu \mathrm{m}$ containing the prelimbic (PL) and infralimbic (IL) prefrontal cortex (from bregma 1.98 $\mathrm{mm}$ to $1.54 \mathrm{~mm}$ ) and the basolateral amygdala (BLA) (from bregma $-1.22 \mathrm{~mm}$ to -1.82 $\mathrm{mm}$ ) were obtained using a microtome. Brain slices were stored in a solution of $5 \%$ sucrose PB 0.1M. Free floating slices were rinsed in PB $0.1 \mathrm{M}$ and after blocked in a solution containing 3\% donkey serum and 0.3\% Triton X-100 in PB 0.1M (DS-T-PB) during $2 \mathrm{~h}$ at room temperature. Slices were incubated overnight with the primary antibody anti-c-Fos in DS-T-PB at $4^{\circ} \mathrm{C}$ (1:500, rabbit, Santa Cruz Biotechnology) (sc7202). Next day, after three rinses with PB $0.1 \mathrm{M}$, slices were incubated with the secondary antibody AlexaFluor-555 donkey anti-rabbit (1:500, Life Technologies) at room temperature for $2 \mathrm{~h}$ in DS-T-PB. Then, slices were rinsed three times and mounted with Mowiol onto glass slides coated with gelatin. 


\subsection{Immunofluorescence Image Analysis}

The stained sections were analyzed at 10x objective using a Leica DMR microscope (Leica Microsystems, Wetzlar, Germany) equipped with a digital camera Leica DFC 300FX (Leica Microsystems). For PL and IL analysis, a $430 \mu \mathrm{m}$ sided square region of interest (ROI) was delimited for quantification. The expression of cFos in the images was quantified using the ImageJ analysis software. The option "particle counting” under a fixed threshold configuration was used to detect cFos positive cells. For all areas, 4 images per mice were quantified. Data are expressed as density of cFos positive cells per mm² (6-9 mice per each experimental condition).

\subsection{Ballistic labeling with the fluorescent dye DiI}

Mice were deeply anesthetized after the last cued fear extinction session by ip injection $(0.2 \mathrm{ml} / 10 \mathrm{~g}$ body weight $)$ of a mixture of ketamine $(100 \mathrm{mg} / \mathrm{kg})$ and xylazine $(20 \mathrm{mg} / \mathrm{kg})$ prior to rapid intracardiac perfusion. Mice were perfused with $10 \mathrm{ml}$ of PBS $0.1 \mathrm{M}, \mathrm{pH}$ 7.5 followed by $40 \mathrm{ml}$ of 4\% (PFA) in PB 0.1M. Brains were postfixed in 4\% PFA for 10 min. Then, brains were kept in PBS 0.1M for $12 \mathrm{~h}$. Brain coronal sections (100 $\mu \mathrm{m})$ containing the amygdala (from bregma $-1.22 \mathrm{~mm}$ to $-1.82 \mathrm{~mm}$ ) were obtained by using a vibratome (Leica VT 1000 S) and kept in PBS 0.1M until fluorescent labeling processing. Brain slices were labeled by ballistic delivery of fluorescent dye DiI (Molecular Probes) using a gene gun apparatus (Helios Gene Gun System, Bio-Rad) as described previously (Gan et al, 2009) and postfixed with PFA for $4 \mathrm{~h}$ at room temperature to further preserve structures and to allow the diffusion of the dye DiI. Sections were placed on microscope gelatine-coated slides and coverslipped with mounting medium (Mowiol). Images were acquired using a confocal microscope (Leica TCS Sp5 STED) with a glycerol immersion 
lens (63X/1.30). Individual pyramidal neurons from the BLA were chosen for spine analysis based on several criteria, as previously described (Saravia et al, 2017). Briefly, (i) there was minimal or no overlap with other labeled cells to ensure that processes from different cells would not be confused, (ii) at least 3 primary dendrites needed to be visible for cells to be used for analysis and (iii) distal dendrites (from secondary dendrites to terminal dendrites) were examined. In addition, we chose only one apical or basal dendrite per neuron and quantified a minimum of 6 dendrites per animal. To calculate spine density, a minimum dendrite length of $20 \mu \mathrm{m}$ long was required. All images of dendrites were taken at different $\mathrm{z}$ levels $(0.13 \mu \mathrm{m}$ depth intervals $)$ to examine the morphology of dendritic spines. Reconstruction of dendrites and spine classification was performed using the IMARIS software (Bitplane). Protrusions from dendrites were classified into 5 types based on their morphology: class 1 or stubby protuberances were $0.5 \mu \mathrm{m}$ in length, lacked a large spine head, and did not appear to have a neck; class 2, or mushroom-shaped spines were between 0.5 and $1.25 \mu \mathrm{m}$ in length and were characterized by a short neck and large spine head; class 3, or thin spines ranged between 1.25 and $3.0 \mu \mathrm{m}$ and had elongated spine necks with small heads; class 4 , or filipodia were 1.0 and $2.5 \mu \mathrm{m}$ in length and were characterized by a large neck without spine head; and class 5 or branched spine ranged between 1.25 and $3.0 \mu \mathrm{m}$ and had elongated spine necks with 2 or more spine heads. Quantification of dendritic spine densities was performed in blind conditions.

\subsection{Data Analysis}

Data were analyzed by using unpaired Student t-test, two-way ANOVA or two-way ANOVA with repeated measures followed by subsequent post hoc analysis (Fisher LSD) when required. The Pearson correlation coefficient was used to analyse the relationship 
between cFos expression and freezing values. A p value $<0.05$ was used to determine statistical significance. The statistical analysis was performed using STATISTICA (StatSoft) software. 


\section{Results}

\subsection{Adolescent THC treatment does not modify fear extinction in adulthood}

Adolescent male mice were treated with increasing doses of THC during 15 days (PND 35-39: $3 \mathrm{mg} / \mathrm{kg}$, PND 40-44: $6 \mathrm{mg} / \mathrm{kg}$, and PND 45-49: $12 \mathrm{mg} / \mathrm{kg}$ ). Body weight was daily evaluated along THC treatment. When mice reached adulthood, the effects of adolescent THC administration on fear memory processing were analyzed (Fig. 1A). Possible changes in locomotor activity and anxiety-like behaviour were also examined (Fig. 1A). The weight gain of mice treated with THC was lower than those exposed to vehicle (Fig. 1B), as previously reported (Rubino et al., 2008; Stopponi et al., 2014; Scherma et al, 2016). An anxiogenic-like effect induced by a high dose of THC could explain the changes in body weight. Indeed, one week after the finishing of the treatment, a higher level of anxiety-like behaviour in adolescent rodents exposed to THC compared to controls was found in the elevated plus maze (EPM) test in previous studies (Stopponi et al., 2014). Non-specific inhibition of ingestion, secondary to the sedative effects of THC could also be involved in this effect on body weight. Locomotor activity $(p=0.96$, Student t test) (Fig. 1C) and anxiety-like responses ( $\mathrm{p}=0.44$, Student $\mathrm{t}$ test) (Fig. 1D) (Fig. S2A) of adult mice were not affected by the chronic treatment with THC. The administration of THC did not modify the acquisition of fear memory as shown by similar freezing behaviour in both contextual ( $\mathrm{p}=0.94$, Student $\mathrm{t}$ test) and cued $(\mathrm{p}=0.78$, Student t test) fear conditioning tests (Fig. $1 E$ and $F$ (Session1)). In addition, cued fear extinction was similar in adult animals exposed to THC or vehicle (Fig. 1F). Thus, two-way ANOVA of repeated measures showed no interaction between THC treatment and day $\left(\mathrm{F}_{5,130}=1.22, \mathrm{p}=0.30\right)($ Fig. $1 F)$.

In an additional experiment, fear memory processing was not modified either in female mice (Fig. S1), in spite of the different sensitivity observed in several behavioural 
responses after treatment with cannabinoid agonists between sexes (Rubino and Parolaro, 2011). Our results indicate that THC administration during adolescence does not alter locomotion, anxiety and fear memory processing in adult male and female mice.

\subsection{Concomitant THC and stress exposure during adolescence impairs fear extinction in adulthood}

Cannabis consumption and environmental factors, such as stressful events, often coexist during adolescence and these factors could exacerbate the detrimental effects of cannabis use in adulthood. To test this hypothesis, we evaluated the consequences of simultaneous adolescent THC and stress exposure on locomotion, anxiety-like behaviour and fear memory processing in adult male mice. For this purpose, one stressor was applied at the end of each THC exposure period as shown in Fig. 2A. Two additional stress exposures were applied before behavioural evaluation (Fig. 2A). Adolescent mice receiving THC, independently of stress exposure, showed again a significant resistance to gain weight (Fig. 2B). Locomotor activity during adulthood was not modified by THC treatment, stress exposure or by the interaction of both factors (Fig. 2C). Mice that underwent stress, independently of THC exposure, showed an increase in anxiety-like behaviour. Thus, adolescent stressed mice spent significantly less time in open arms in the EPM (stress effect: $F_{1,60}=4.17, \mathrm{p}<0.05$ ) (Fig. $2 D$ ), without significant interaction between THC and stress. This effect was not due to differences in the total number of entries (Fig. S2B). The acquisition of fear memory was not altered in adult mice exposed to stress, THC or both factors simultaneously during adolescence. Thus, freezing levels were similar in both contextual $\left(\mathrm{F}_{1,58}=2.62\right.$, NS) and cued $\left(\mathrm{F}_{1,59}=2.69\right.$, NS) fear conditioning tests (Fig. $2 E$ and $F(\mathrm{~S} 1))$. Notably, concomitant THC and stress exposure impaired cued fear 
extinction (Fig. 2F). Two-way ANOVA with repeated measures revealed a significant interaction between THC and stress $\left(\mathrm{F}_{1,59}=9.38, \mathrm{p}<0.01\right)$, without other two-way or threeway interactions. Two-way ANOVA analyzed for each individual session revealed a significant interaction between stress and THC from session 4 to session $6\left(F_{1,59}=10.26\right.$, $\mathrm{p}<0.01$ at session 4; $\mathrm{F}_{1,59}=5.45, \mathrm{p}<0.05$ at session $5 ; \mathrm{F}_{1,59}=15.56, \mathrm{p}<0.01$ at session 6 ). Subsequent post hoc analysis showed higher freezing levels in mice simultaneously exposed to THC and stress in comparison with those treated with THC ( $<<0.05$ at session 5; $p<0.01$ at session 4 and 6), exposed to stress ( $p<0.01$ at session 4, 5 and 6 ), and vehicle non-stress mice ( $\mathrm{p}<0.01$ at session 4 and 6; $\mathrm{p}<0.05$ at session 5) (Fig. 2F). In agreement, freezing levels expressed as AUC (Fig. 2G) were higher in mice exposed simultaneously to THC and stress in comparison with the other experimental conditions, as revealed by two-way ANOVA (interaction THC $\mathrm{x}$ stress: $\mathrm{F}_{1,59}=8.51, \mathrm{p}<0.01$ ) and post hoc analysis $(\mathrm{p}<0.01)$. We next evaluated whether the impairment in cued fear extinction could be related to an alteration of CB1R levels in the amygdala. CB1R in this brain region plays a crucial role in the modulation of fear processing (Gunduz-Cinar et al., 2013). Western blot analysis showed no differences in total CB1R levels in the amygdala of adult mice exposed to THC and/or stress during the adolescent period (interaction THC x stress: $\mathrm{F}_{1,19}=1.99, \mathrm{p}=0.17$ ) (Fig. S3). This result suggests that, under our experimental conditions, impairment in fear extinction is not influenced by a reduced expression of CB1R in the amygdala of adult animals. However, changes in the efficacy or efficiency of the receptor binding can occur independently from the status of the receptor density, contributing to the observed phenotype.

To elucidate whether immature brain represents a period of development more susceptible to the effects of THC and stress, we compared the consequences of exposing the same type of stressors and THC treatment during adolescence and adulthood period 
(Fig. S4A). For this purpose, changes in behaviour in adult male animals were evaluated exactly 20 days following the final day of THC administration (Fig. S4A), as previously studied after exposure during the adolescent period. Notably, no differences in cued fear processing were observed between the different experimental groups exposed to THC and/or stress in adulthood period (Fig. S4B and $C$ ), suggesting that adolescence is a sensitive window for the harmful consequences of concomitant THC and stress exposure.

\subsection{Concomitant THC and stress exposure during adolescence increases corticosterone levels following cued fear conditioning session}

Stress during early-stages can impair fear extinction by modulation of glucocorticoid activity (Green et al, 2011). Therefore, we next evaluated possible changes in plasma corticosterone levels due to THC and stress exposure during adolescence at different time points (Fig. 3A). Corticosterone levels measured immediately after restraint stress exposure were significantly increased in stressed mice (stress effect: $F_{1,27}=300.43$, $\mathrm{p}<0.01$ ) (Fig. 3B), without influence of THC treatment (Veh-non stress: $68.38 \pm 8.8$ ng/ml; Veh-stress: $333.57 \pm 11.57$ ng/ml; THC-non stress: $82.25 \pm 19.44$; THC-stress: $365.38 \pm 19.12 \mathrm{ng} / \mathrm{ml})$. Basal levels of corticosterone after previous THC and stress exposure (PND 67) were unaltered in the different experimental groups (Fig. S5). Corticosterone levels measured 30 min after fear conditioning training were higher than in basal conditions, but remained similar in all experimental groups (Fig. 3C). Interestingly, previous simultaneous exposure to THC and stress increased plasma corticosterone 30 min after cued fear conditioning testing, as shown by two-way ANOVA (interaction treatment $\mathrm{x}$ stress: $\mathrm{F}_{1,27}=5.76, \mathrm{p}<0.05$ ) (Fig. $3 D$ ), and post hoc analysis $(\mathrm{p}<0.01$, THC-stress $(132.88 \pm 8.06 \mathrm{ng} / \mathrm{ml})$ versus THC-non stress mice $(89.13 \pm 5.33$ 
ng/ml) (Fig. 3D). This imbalance of corticosterone activity was temporal since similar levels were again observed after the fifth cued extinction session (Fig. 3E). These results suggest that glucocorticoid sensitization after cued fear conditioning may contribute to the extinction deficits revealed in mice exposed to THC and stress during the adolescent period.

\subsection{Impaired fear extinction induced by concomitant THC and stress exposure is associated with reduced activity of the infralimbic prefrontal cortex and the basolateral amygdala}

To identify the brain areas responsible for the resistance to fear extinction induced by simultaneous THC and stress exposure, we analyzed the possible activation of brain regions closely involved in the extinction circuit by using cFos immunofluorescence. cFos expression in the PL, IL and BLA was evaluated $2 \mathrm{~h}$ after the last cued fear extinction session, as behavioural alteration remained present at this time point. cFos expression in the PL was not altered in the different experimental groups $\left(\mathrm{F}_{1,20}=1.05\right.$, NS) (Fig. S6). Interestingly, IL activation was reduced by the previous concomitant exposure to THC and stress $(\mathrm{p}<0.01)$, as shown by two-way ANOVA (interaction treatment $\mathrm{x}$ stress $\mathrm{F}_{1,32}=6.70, \mathrm{p}<0.05$ ) (Fig. $4 A$ and $B$ ). Values of $\mathrm{cFos}$ expression in the IL for the different experimental groups were the following: Veh-non stress: $247.10 \pm 16.10$ cFos + cells/mm²; Veh-stress: $249.47 \pm 25.36$ cFos + cells $/ \mathrm{mm}^{2}$; THC-non stress: $253.76 \pm 8.92$ cFos+ cells/mm²; THC-stress: $171.00 \pm 12.16$ cFos + cells $/ \mathrm{mm}^{2}$ ). Likewise, simultaneous THC and stress adolescent exposure reduced the activity of the BLA as revealed by twoway ANOVA (interaction treatment $x$ stress $F_{1,28}=4.51, p<0.05$ ), and post hoc analysis $(\mathrm{p}<0.05)$ (Fig. $4 C$ and $D$ ). Values of $\mathrm{cFos}$ expression in the BLA for the different 
experimental groups were the following: Veh-non stress: $144.03 \pm 12.21$ cFos+ cells/mm²; Veh-stress: $149.97 \pm 14.12$ cFos + cells $/ \mathrm{mm}^{2}$; THC-non stress: $138.60 \pm 7.98$ cFos+ cells $/ \mathrm{mm}^{2}$; THC-stress: $108.83 \pm 9.75$ cFos + cells $/ \mathrm{mm}^{2}$ ). These results show that the impaired fear extinction induced by adolescent THC and stress exposure is associated with reduced activity of the IL and the BLA, which are key regions involved in the extinction of aversive memories. Indeed, a significant negative correlation $(\mathrm{p}<0.05)$ between fear memory (freezing values) and IL activity (density of cFos+ cells) was observed (Fig. $4 E$ ), whereas a clear tendency $(\mathrm{p}=0.058)$ was revealed in the BLA (Fig. $4 F$ ). In an additional experiment cFos expression was analyzed in the BLA and the IL in adult mice exposed to THC and stress during the adolescence just before and after the fear conditioning and extinction paradigm. These experiments were carried out in order to investigate whether the reduction in the activity of these brain areas was due to the THC-stress treatment or if these changes were influenced by the dynamic interplay among THC, stress and fear conditioning/extinction. Simultaneous THC and stress adolescent exposure reduced the activity of the BLA (interaction THC $x$ stress: $F_{1,23}=9.52, p<0.05$ ) (Fig. S7A) and the IL (interaction THC x stress: $\mathrm{F}_{1,22}=5.66, \mathrm{p}<0.05$ ) (Fig S7B) after cued fear extinction, as previously reported. Interestingly, cFos expression was higher in adult animals exposed to THC and stress during the adolescence that did not undergo fear conditioning/extinction in both the BLA $(\mathrm{p}<0.05)$ (Fig. S7A) and the IL $(\mathrm{p}<0.01)$ (Fig $\mathrm{S} 7 \mathrm{~B}$ ) in comparison with mice that followed the behavioural paradigm. These results suggest that the reduced activity observed in the BLA and the IL depends on the dynamic effect of THC-stress exposure followed by fear conditioning and extinction behavioural testing. 


\subsection{Impaired fear extinction induced by concomitant THC and stress exposure is associated with structural plasticity alterations in the basolateral amygdala}

Stress has been reported to alter structural plasticity in the amygdala (Leuner and Shors, 2013), affect fear extinction (Maren and Holmes, 2016), and THC exposure during adolescence can induce changes in dendritic spines in several brain regions (Rubino and Parolaro, 2016). Given the crucial role played by the BLA in fear extinction, we investigated whether concomitant exposure to THC and stress could affect structural plasticity in this brain area. Mice were sacrificed after the last cued extinction session and brains were processed for ballistic delivery to label whole neurons with the dye DiI. Total dendritic spine density of BLA pyramidal neurons was not modified by THC, stress or by the combination of both factors (Fig. 5A). Dendritic spines are dynamic and can be classified into different categories depending on their morphology (stubby, mushroom, thin, branched and filopodia). Density of mushroom (mature) spines was reduced in adolescent mice treated with THC (independently of stress exposure) $(\mathrm{p}<0.01)$, as revealed by two-way ANOVA (treatment effect: $\mathrm{F}_{1,23}=9.35, \mathrm{p}<0.01$ ) (Fig. $5 B$ and $C$ ). THC treatment increased the density of thin (immature) spines (treatment effect: $\mathrm{F}_{1,23}=7.43 \mathrm{p}<0.05$ ) (Fig. $5 B$ and $C$ ). Notably, this effect was mainly due to the concomitant exposure to THC and stress as revealed by post hoc comparisons. Thus, THC-stress mice showed higher density of thin spines in comparison with THC-non stress $(\mathrm{p}<0.05)$, vehicle-stress $(\mathrm{p}<0.05)$, and vehicle-non stress $(\mathrm{p}<0.01)$ mice (Fig. $5 B$ and $C)$. These data suggest that changes in the density of immature spines in the BLA might be involved in the impairment of fear extinction associated with the combination of THC and stress exposure during the adolescence. 


\section{Discussion}

To the best of our knowledge, this is the first study demonstrating synergistic detrimental effects of simultaneous adolescent THC and stress exposure on the extinction of aversive memory in adulthood. Notably, impaired fear extinction was associated with a temporal imbalance of plasmatic corticosterone levels, decreased activity of key regions involved in fear regulation, such as the IL and the BLA, and changes in structural plasticity revealed by increased immature dendritic spines density in pyramidal neurons of the BLA.

The administration of THC during adolescence in male and female mice did not induce any modification in locomotor activity, anxiety-like responses, and fear regulation later in life. Controversial results have been previously reported after adolescent THC exposure since general anxiety by using EPM was not modified in adult rats (Rubino et al, 2008; Cadoni et al, 2015), whereas an anxiogenic-like effect was revealed in adult CD1 mice (Murphy et al, 2017). In agreement with our data, no lasting effects by adolescent THC exposure were previously observed in aversive memory as revealed by the lack of deficits in passive avoidance task (Rubino et al, 2009a; Rubino et al, 2009b) or cued and contextual fear conditioning (Ballinger et al, 2015). However, a long-lasting impairment of fear conditioning has been described after chronic administration of the synthetic cannabinoid WIN 55,212-2 during adolescence (Gleason et al, 2012; TomasRoig et al, 2017), which has a higher intrinsic activity on cannabinoid receptors than THC (Kuster et al, 1993). On the other hand, repeated adolescent CB1R antagonism induced greater contextual fear memory recall in adult female, but not male, rats in comparison with control animals (Simone et al, 2018b). Fear extinction, which has not been 
previously evaluated, was not affected in either male or female mice by adolescent THC treatment under our experimental conditions.

Although the exact cause of most psychiatric illnesses is not well known, it is becoming clear that many of these conditions are caused by the combination of genetic and environmental factors or by the association between different environmental events (Caspi and Moffit, 2006; Rubino and Parolaro, 2016). Stress has a critical role in the development of many psychiatric conditions including trauma-related disorders (Maren and Holmes, 2016). Fear extinction deficits have been reported following exposure to various types of stressors in either animal models (Maren and Holmes, 2016) or humans (Hartley et al, 2014). Thus, adolescent rodents exposed to chronic stress usually show impaired extinction when tested as adults (Judo et al, 2010; Ishikawa et al, 2012; ToledoRodriguez et al, 2012; Skelly et al, 2015), although different results have been reported depending on the sex and the modality, chronicity and precise timing of stress exposure during adolescence (Morrissey et al, 2011; McCormick et al, 2013; Schayek and Maroun, 2015; Deng et al, 2017). We found that adolescent stress exposure enhanced anxiety-like behaviour, but did not modify fear conditioning in adult mice. In contrast, concomitant adolescent THC and stress exposure induced an impairment of fear extinction in adulthood without affecting the acquisition of fear memory or general anxiety. Notably, the effect of this THC/stress exposure was age-dependent as revealed by the lack of deficits in fear extinction when the same exposure was performed directly in adulthood. Impaired extinction was not observed by the individual exposition to each factor suggesting synergistic detrimental consequences of adolescent cannabis and stressful events. In this regard, chronic adolescent treatment with THC induced a deficit in cued fear conditioning only in adult mice with a mutation in disrupted-in-schizophrenia 1 gene 
(Ballinger et al, 2015), although the influence of this genetic and environmental interaction in fear extinction was not evaluated in this study.

Animals simultaneously exposed to THC and stress during adolescence showed a temporal imbalance of plasmatic corticosterone levels in the adult period as revealed by the increase of this hormone following the cued fear conditioning session. Although the association between glucocorticoids and fear extinction is complex, this enhancement of the hypothalamic-pituitary-adrenal (HPA) axis activity could participate in the extinction deficits and changes in structural plasticity revealed in our study. Thus, a single exposure to immobilization stress in adult male mice activates the HPA axis by increasing corticosterone plasma levels (Andero et al, 2011). A week after this stress exposure mice present impaired cued-fear extinction and enhanced levels of corticosterone after both fear acquisition and fear extinction (Sawamura et al, 2016). Moreover, acute corticosterone administration in adult rats induced dendritic hypertrophy of BLA neurons and enhanced anxiety 12 days after the treatment (Mitra and Sapolsky, 2008; Kim et al, 2014).

IL and BLA are key structures involved in the neurobiological substrate underlying fear extinction (Sierra-Mercado et al, 2011). IL facilitates the activation of the subpopulation of BLA neurons directly involved in fear extinction (Herry et al, 2008). Several studies evaluating immediately-early gene (IEG) expression conclude that impaired fear extinction is associated with reduced activity of the cortico-amygdala circuit (Herry and Mons, 2004; Holmes and Singewald, 2013), while increased IEG levels in those brain areas is related to a complete extinction of conditioned fear (Herry and Mons, 2004; Flores et al, 2014). Notably, our data reveal a reduced cFos expression in the IL and the BLA in adult mice simultaneously exposed to THC and stress during the adolescent 
period suggesting the existence of a long-lasting dysregulation of the fear circuit. In contrast, PL activity was not modified consistent with a role for this brain area in fear expression, but not extinction (Sierra-Mercado et al, 2011). Besides these modifications on brain region activity, we found structural plasticity changes in pyramidal neurons of the BLA in adulthood as a consequence of adolescent THC treatment, alone or in combination with stress. A decrease of mushroom (matures) dendritic spines was revealed in mice previously exposed to THC congruent with previous studies showing reduced spine density in the dentate gyrus of the hippocampus (Rubino et al, 2009b) and the prefrontal cortex (Rubino et al, 2015) of adult male and female rats, respectively. Interestingly, simultaneous adolescent THC and stress exposure induced an increase of thin (immatures) spines in pyramidal neurons of the BLA in adult mice. These dendritic morphology modifications in BLA neurons associated with the decreased activity of this nucleus could be responsible of the deficits in the extinction of aversive memories. In agreement, fear extinction deficits following acute stress have been related to dendritic retraction in pyramidal neurons of both BLA (Maroun et al, 2013) and IL (Moench et al, 2016).

In summary, our data show lasting neurobiological changes associated with resistance to fear extinction due to concomitant adolescent THC and stress exposure. This study identifies a potential social group highly vulnerable to develop anxiety disorders characterized by pathological fear after cannabis consumption, which contains multitude bioactive compounds (ElSohly et al, 2017). Although our study was performed using pure THC, significantly higher THC concentrations in cannabis derived extracts over the years have been reported (Cascini et al, 2012; ElSohly et al, 2016), which could cause an increase of the total amount of THC consumed. This increase poses higher risk of cannabis use, particularly among adolescents (ElSohly et al, 2016). Taking into account 
the high rate of cannabis intake during this period which usually entails stressful events, the combination of both factors may increase the risk to suffer anxiety disorders in the adult period. Indeed, the exposure to stressful events during adolescence that often occur in cannabis consumers may represent an important risk factor to suffer anxiety disorders in adulthood. 


\section{Acknowledgements}

This work was supported by “Plan Nacional sobre Drogas” (\#2014I019) and “Ministerio de Economía y Competitividad” (SAF2017-85299-R) to FB, the "Generalitat de Catalunya-AGAUR" (\#2014-SGR-1547), ICREA-Acadèmia (2015) and "Ministerio de Economía y Competitividad” (\#SAF2017-84060-R) to RM, NARSAD Young Investigator Grant \#22434, Ramón y Cajal program RYC2014-15784, RETOS-MINECO SAF2016-76565-R and FEDER funds to RA, and "Ministerio de Economía y Competitividad” (SAF2014-53876R) and “Generalitat de Catalunya” (SGR 2014-1020) to AA. Rocio Saravia is a recipient of a predoctoral fellowship from the "Instituto de Salud Carlos III”. We thank Raquel Martín for expert technical assistance and África Flores for her assistance in performing pilot behavioural determinations.

\section{Conflict of interest}

The authors declare that there are no conflicts of interest. 


\section{References}

Andero R, Heldt SA, Ye K, Liu X, Armario A, Ressler KJ. 2011. Effect of 7,8dihydroxyflavone, a small-molecule TrkB agonist, on emotional learning. Am J Psychiatry 168, 163-172.

Ballinger MD, Saito A, Abazyan B, Taniguchi Y, Huang CH, Ito K, Zhu X, Segal H, Jaaro-Peled H, Sawa A, Mackie K, Pletnikov MV, Kamiya A. 2015. Adolescent cannabis exposure interacts with mutant DISC1 to produce impaired adult emotional memory. Neurobiol Dis 82, 176-184.

Bilkei-Gorzo A, Erk S, Schürmann B, Mauer D, Michel K, Boecker H, Scheef L, Walter H, Zimmer A. 2012. Dynorphins regulate fear memory: from mice to men. J Neurosci 32, 9335-9343.

Biscaia M, Marín S, Fernández B, Marco EM, Rubio M, Guaza C, Ambrosio E, Viveros MP. (2003). Chronic treatment with CP 55,940 during the peri-adolescent period differentially affects the behavioural responses of male and female rats in adulthood. Psychopharmacology (Berl) 170, 301-308.

Bortolato M, Bini V, Frau R, Devoto P, Pardu A, Fan Y, Solbrig MV. (2014). Juvenile cannabinoid treatment induces frontostriatal gliogenesis in Lewis rats. Eur Neuropsychopharmacol 24, 974-985.

Cadoni C, Simola N, Espa E, Fenu S, Di Chiara G. 2015. Strain dependence of adolescent Cannabis influence on heroin reward and mesolimbic dopamine transmission in adult Lewis and Fischer 344 rats. Addict Biol 20, 132-142. 
Cascini F, Aiello C, Di Tanna G. 2012. Increasing delta-9-tetrahydrocannabinol ( $\Delta-9-$ THC) content in herbal cannabis over time: systematic review and meta-analysis. Curr Drug Abuse Rev 5, 32-40

Caspi A, Moffitt TE. 2006. Gene-environment interactions in psychiatry: joining forces with neuroscience. Nat Rev Neurosci 7, 583-590.

Deng JH, Yan W, Han Y, Chen C, Meng SQ, Sun CY, Xu LZ, Xue YX, Gao XJ, Chen N, Zhang FL, Wang YM, Shi J, Lu L. 2017. Predictable Chronic Mild Stress during Adolescence Promotes Fear Memory Extinction in Adulthood. Sci Rep 7(1):7857.

ElSohly MA, Radwan MM, Gul W, Chandra S, Galal A. 2017. Phytochemistry of Cannabis sativa L. Prog Chem Org Nat Prod 103, 1-36.

ElSohly MA, Mehmedic Z, Foster S, Gon C, Chandra S, Church JC. 2016. Changes in Cannabis Potency Over the Last 2 Decades (1995-2014): Analysis of Current Data in the United States. Biol Psychiatry 79, 613-619.

Fernández-Ruiz J, Berrendero F, Hernández ML, Ramos JA. 2000. The endogenous cannabinoid system and brain development. Trends Neurosci 23, 14-20.

Flores Á, Valls-Comamala V, Costa G, Saravia R, Maldonado R, Berrendero F. 2014. The hypocretin/orexin system mediates the extinction of fear memories. Neuropsychopharmacology 39, 2732-2741.

Gan WB, Grutzendler J, Wong RO, Lichtman JW. 2009. Ballistic delivery of dyes for structural and functional studies of the nervous system. Cold Spring Harb Protoc 2009(4):pdb.prot5202.

Gibaldi M, Perrier D. 1975. The trapezoid rule. In: Gibaldi M, Perier D (eds), pp. Pharmacokinetics. Marcel Dekker: New York. pp. 293-296. 
Gleason KA, Birnbaum SG, Shukla A, Ghose S. 2012. Susceptibility of the adolescent brain to cannabinoids: long-term hippocampal effects and relevance to schizophrenia. Transl Psychiatry 2:e199.

Green MK, Rani CS, Joshi A, Soto-Piña AE, Martinez PA, Frazer A, Strong R, Morilak DA. 2011. Prenatal stress induces long term stress vulnerability, compromising stress response systems in the brain and impairing extinction of conditioned fear after adult stress. Neuroscience 192, 438-451.

Gunduz-Cinar O, Hill MN, McEwen BS, Holmes A. 2013. Amygdala FAAH and anandamide: mediating protection and recovery from stress. Trends Pharmacol Sci 34, 637-644.

Harkany T, Guzmán M, Galve-Roperh I, Berghuis P, Devi LA, Mackie K. 2007. The emerging functions of endocannabinoid signaling during CNS development. Trends Pharmacol Sci 28, 83-92.

Hartley CA, Gorun A, Reddan MC, Ramirez F, Phelps EA. 2014. Stressor controllability modulates fear extinction in humans. Neurobiol Learn Mem 113, 149-156.

Herry C, Mons N. 2004. Resistance to extinction is associated with impaired immediate early gene induction in medial prefrontal cortex and amygdala. Eur J Neurosci 20, 781790.

Herry C, Ciocchi S, Senn V, Demmou L, Müller C, Lüthi A. 2008. Switching on and off fear by distinct neuronal circuits. Nature 454, 600-606.

Higuera-Matas A, Ucha M, Ambrosio E. 2015. Long-term consequences of perinatal and adolescent cannabinoid exposure on neural and psychological processes. Neurosci Biobehav Rev 55, 119-146. 
Holmes A, Singewald N. 2013. Individual differences in recovery from traumatic fear. Trends Neurosci 36, 23-31.

Ishikawa S, Saito Y, Yanagawa Y, Otani S, Hiraide S, Shimamura K, Matsumoto M, Togashi H. 2012. Early postnatal stress alters extracellular signal-regulated kinase signaling in the corticolimbic system modulating emotional circuitry in adult rats. Eur $\mathrm{J}$ Neurosci 35, 135-145.

Judo C, Matsumoto M, Yamazaki D, Hiraide S, Yanagawa Y, Kimura S, Shimamura K, Togashi H. 2010. Early stress exposure impairs synaptic potentiation in the rat medial prefrontal cortex underlying contextual fear extinction. Neuroscience 169, 1705-1714.

Keeley RJ, Trow J, Bye C, McDonald RJ. 2015. Part II: Strain- and sex-specific effects of adolescent exposure to THC on adult brain and behaviour: Variants of learning, anxiety and volumetric estimates. Behav Brain Res 288, 132-152.

Ketcherside A, Filbey FM. 2015. Mediating processes between stress and problematic marijuana use. Addict Behav 45, 113-118.

Kim H, Yi JH, Choi K, Hong S, Shin KS, Kang SJ. 2014. Regional differences in acute corticosterone-induced dendritic remodeling in the rat brain and their behavioral consequences. BMC Neurosci 15:65.

Klug M, van den Buuse M. 2012. Chronic cannabinoid treatment during young adulthood induces sex-specific behavioural deficits in maternally separated rats. Behav Brain Res 233, 305-313.

Kuster JE, Stevenson JI, Ward SJ, D'Ambra TE, Haycock DA. 1993. Aminoalkylindole binding in rat cerebellum: selective displacement by natural and synthetic cannabinoids. J Pharmacol Exp Ther 264, 1352-1363. 
Lee JY, Brook JS, Finch SJ, Brook DW. 2017. Trajectories of cannabis use beginning in adolescence associated with symptoms of posttraumatic stress disorder in the mid-thirties. Subst Abus Aug 3:1-7 [Epub ahead of print]

Leuner B, Shors TJ. 2013. Stress, anxiety, and dendritic spines: what are the connections? Neuroscience 251, 108-119.

Llorente-Berzal A, Fuentes S, Gagliano H, López-Gallardo M, Armario A, Viveros MP, Nadal R. 2011. Sex-dependent effects of maternal deprivation and adolescent cannabinoid treatment on adult rat behaviour. Addict Biol 16, 624-637.

Llorente-Berzal A, Puighermanal E, Burokas A, Ozaita A, Maldonado R, Marco EM, Viveros MP. 2013. Sex-dependent psychoneuroendocrine effects of THC and MDMA in an animal model of adolescent drug consumption. PLoS One 8(11):e78386.

Long LE, Chesworth R, Huang XF, McGregor IS, Arnold JC, Karl T. 2013. Transmembrane domain Nrg1 mutant mice show altered susceptibility to the neurobehavioural actions of repeated THC exposure in adolescence. Int $\mathrm{J}$ Neuropsychopharmacol 16, 163-175.

McCormick CM, Mongillo DL, Simone JJ. 2013. Age and adolescent social stress effects on fear extinction in female rats. Stress 16, 678-688.

Malone DT, Hill MN, Rubino T. 2010. Adolescent cannabis use and psychosis: epidemiology and neurodevelopmental models. Br J Pharmacol 160, 511-522.

Maren S, Holmes A. 2016. Stress and Fear Extinction. Neuropsychopharmacology 41, 58-79. 
Maroun M, Ioannides PJ, Bergman KL, Kavushansky A, Holmes A, Wellman CL. 2013. Fear extinction deficits following acute stress associate with increased spine density and dendritic retraction in basolateral amygdala neurons. Eur J Neurosci 38, 2611-2620.

Mitra R, Sapolsky RM. 2008. Acute corticosterone treatment is sufficient to induce anxiety and amygdaloid dendritic hypertrophy. Proc Natl Acad Sci U S A 105, 55735578.

Moench KM, Maroun M, Kavushansky A, Wellman CL. 2016. Alterations in neuronal morphology in infralimbic cortex predict resistance to fear extinction following acute stress. Neurobiol Stress 3, 23-33.

Morrissey MD, Mathews IZ, McCormick CM. 2011. Enduring deficits in contextual and auditory fear conditioning after adolescent, not adult, social instability stress in male rats. Neurobiol Learn Mem 95, 46-56.

Murphy M, Mills S, Winstone J, Leishman E, Wager-Miller J, Bradshaw H, Mackie K. 2017. Chronic Adolescent 49 -Tetrahydrocannabinol Treatment of Male Mice Leads to Long-Term Cognitive and Behavioral Dysfunction, Which Are Prevented by Concurrent Cannabidiol Treatment. Cannabis Cannabinoid Res 2, 235-246.

Na ES, Nelson ED, Adachi M, Autry AE, Mahgoub MA, Kavalali ET, Monteggia LM. 2012. A mouse model for MeCP2 duplication syndrome: MeCP2 overexpression impairs learning and memory and synaptic transmission. J Neurosci 32, 3109-3117.

O'Tuathaigh CM, Hryniewiecka M, Behan A, Tighe O, Coughlan C, Desbonnet L, Cannon M, Karayiorgou M, Gogos JA, Cotter DR, Waddington JL. 2012. Chronic adolescent exposure to $\Delta$-9-tetrahydrocannabinol in COMT mutant mice: impact on psychosis-related and other phenotypes. Neuropsychopharmacology 35, 2262-2273. 
Paxinos G, Franklin KBJ. 2001. The mouse brain in stereotaxic coordinates. Academic Press: San Diego, CA, USA.

Quinn HR, Matsumoto I, Callaghan PD, Long LE, Arnold JC, Gunasekaran N, Thompson MR, Dawson B, Mallet PE, Kashem MA, Matsuda-Matsumoto H, Iwazaki T, McGregor IS. 2008. Adolescent rats find repeated Delta(9)-THC less aversive than adult rats but display greater residual cognitive deficits and changes in hippocampal protein expression following exposure. Neuropsychopharmacology 33, 1113-1126.

Realini N, Vigano' D, Guidali C, Zamberletti E, Rubino T, Parolaro D. 2011. Chronic URB597 treatment at adulthood reverted most depressive-like symptoms induced by adolescent exposure to THC in female rats. Neuropharmacology 60(2-3), 235-243.

Renard J, Rushlow WJ, Laviolette SR. 2016. What Can Rats Tell Us about Adolescent Cannabis Exposure? Insights from Preclinical Research. Can J Psychiatry 61, 328-334.

Renard J, Rosen LG, Loureiro M, De Oliveira C, Schmid S, Rushlow WJ, Laviolette SR. 2017. Adolescent Cannabinoid Exposure Induces a Persistent Sub-Cortical HyperDopaminergic State and Associated Molecular Adaptations in the Prefrontal Cortex. Cereb Cortex 27, 1297-1310.

Rubino T, Vigano' D, Realini N, Guidali C, Braida D, Capurro V, Castiglioni C, Cherubino F, Romualdi P, Candeletti S, Sala M, Parolaro D. 2008. Chronic delta 9tetrahydrocannabinol during adolescence provokes sex-dependent changes in the emotional profile in adult rats: behavioral and biochemical correlates. Neuropsychopharmacology 33, 2760-2771.

Rubino T, Realini N, Braida D, Alberio T, Capurro V, Viganò D, Guidali C, Sala M, Fasano M, Parolaro D. 2009a. The depressive phenotype induced in adult female rats by 
adolescent exposure to THC is associated with cognitive impairment and altered neuroplasticity in the prefrontal cortex. Neurotox Res 15, 291-302.

Rubino T, Realini N, Braida D, Guidi S, Capurro V, Viganò D, Guidali C, Pinter M, Sala M, Bartesaghi R, Parolaro D. 2009b. Changes in hippocampal morphology and neuroplasticity induced by adolescent THC treatment are associated with cognitive impairment in adulthood. Hippocampus 19, 763-772.

Rubino T, Parolaro D. 2011. Sexually dimorphic effects of cannabinoid compounds on emotion and cognition. Front Behav Neurosci 5:64.

Rubino T, Prini P, Piscitelli F, Zamberletti E, Trusel M, Melis M, Sagheddu C, Ligresti A, Tonini R, Di Marzo V, Parolaro D. 2015. Adolescent exposure to THC in female rats disrupts developmental changes in the prefrontal cortex. Neurobiol Dis 73, 60-69.

Rubino T, Parolaro D. 2016. The Impact of Exposure to Cannabinoids in Adolescence: Insights From Animal Models. Biol Psychiatry 79, 578-585.

Saravia R, Flores Á, Plaza-Zabala A, Busquets-Garcia A, Pastor A, de la Torre R, Di Marzo V, Marsicano G, Ozaita A, Maldonado R, Berrendero F. 2017. CB1 Cannabinoid receptors mediate cognitive deficits and structural plasticity changes during nicotine withdrawal. Biol Psychiatry 81, 625-634.

Sawamura T, Klengel T, Armario A, Jovanovic T, Norrholm SD, Ressler KJ, Andero R. 2016. Dexamethasone Treatment Leads to Enhanced Fear Extinction and Dynamic Fkbp5 Regulation in Amygdala. Neuropsychopharmacology 41, 832-846.

Schayek R, Maroun M. 2015. Differences in stress-induced changes in extinction and prefrontal plasticity in postweanling and adult animals. Biol Psychiatry 78, 159-166. 
Scherma M, Dessì C, Muntoni AL, Lecca S, Satta V, Luchicchi A, Pistis M, Panlilio LV, Fattore L, Goldberg SR, Fratta W, Fadda P. 2016. Adolescent $\Delta(9)$-Tetrahydrocannabinol Exposure Alters WIN55,212-2 Self-Administration in Adult Rats. Neuropsychopharmacology 41, 1416-1426.

Schneider M. 2008. Puberty as a highly vulnerable developmental period for the consequences of cannabis exposure. Addict Biol 13, 253-263.

Schneider M. 2013. Adolescence as a vulnerable period to alter rodent behavior. Cell Tissue Res 354, 99-106.

Sierra-Mercado D, Padilla-Coreano, Quirk GJ. 2011. Dissociable roles of prelimbic and infralimbic cortices, ventral hippocampus, and basolateral amygdala in the expression and extinction of conditioned fear. Neuropsychopharmacology 36, 529-538.

Silins E, Horwood LJ, Patton GC, Fergusson DM, Olsson CA, Hutchinson DM, Spry E, Toumbourou JW, Degenhardt L, Swift W, Coffey C, Tait RJ, Letcher P, Copeland J, Mattick RP; Cannabis Cohorts Research Consortium. 2014. Young adult sequelae of adolescent cannabis use: an integrative analysis. Lancet Psychiatry 1, 286-293.

Simone JJ, Baumbach JL, McCormick CM. 2018a. Effects of CB1 receptor antagonism and stress exposures in adolescence on socioemotional behaviours, neuroendocrine stress responses, and expression of relevant proteins in the hippocampus and prefrontal cortex in rats. Neuropharmacology 128, 433-447.

Simone JJ, Baumbach JL, McCormick CM. 2018b. Sex-specific effects of CB1 receptor antagonism and stress in adolescence on anxiety, corticosterone concentrations, and contextual fear in adulthood in rats. Int J Dev Neurosci 69,119-131. 
Soria-Gómez E, Busquets-Garcia A, Hu F, Mehidi A, Cannich A, Roux L, Louit I, Alonso

L, Wiesner T, Georges F, Verrier D, Vincent P, Ferreira G, Luo M, Marsicano G. 2015. Habenular CB1 Receptors Control the Expression of Aversive Memories. Neuron 88, 306-313.

Skelly MJ, Chappell AE, Carter E, Weiner JL. 2015. Adolescent social isolation increases anxiety-like behavior and ethanol intake and impairs fear extinction in adulthood: Possible role of disrupted noradrenergic signaling. Neuropharmacology 97, 149-159.

Spechler PA, Orr CA, Chaarani B, Kan KJ, Mackey S, Morton A, Snowe MP, Hudson KE, Althoff RR, Higgins ST, Cattrell A, Flor H, Nees F, Banaschewski T, Bokde ALW, Whelan R, Büchel C, Bromberg U, Conrod P, Frouin V, Papadopoulos D, Gallinat J, Heinz A, Walter H, Ittermann B, Gowland P, Paus T, Poustka L, Martinot JL, Artiges E, Smolka MN, Schumann G, Garavan H; IMAGEN Consortium. 2015. Cannabis use in early adolescence: Evidence of amygdala hypersensitivity to signals of threat. Dev Cogn Neurosci 16, 63-70.

Stockhorst U, Antov MI. 2016. Modulation of Fear Extinction by Stress, Stress Hormones and Estradiol: A Review. Front Behav Neurosci 9,359.

Stopponi S, Soverchia L, Ubaldi M, Cippitelli A, Serpelloni G, Ciccocioppo R. 2014. Chronic THC during adolescence increases the vulnerability to stress-induced relapse to heroin seeking in adult rats. Eur Neuropsychopharmacol 24, 1037-1045.

Sturman DA, Moghaddam B. 2011. The neurobiology of adolescence: changes in brain architecture, functional dynamics, and behavioral tendencies. Neurosci Biobehav Rev 35, 1704-1712. 
Toledo-Rodriguez M, Pitiot A, Paus T, Sandi C. 2012. Stress during puberty boosts metabolic activation associated with fear-extinction learning in hippocampus, basal amygdala and cingulate cortex. Neurobiol Learn Mem 98, 93-101.

Tomas-Roig J, Benito E, Agis-Balboa RC, Piscitelli F, Hoyer-Fender S, Di Marzo V, Havemann-Reinecke U. 2017. Chronic exposure to cannabinoids during adolescence causes long-lasting behavioral deficits in adult mice. Addict Biol 22, 1778-1789.

Zamberletti E, Prini P, Speziali S, Gabaglio M, Solinas M, Parolaro D, Rubino T. 2012. Gender-dependent behavioral and biochemical effects of adolescent delta-9tetrahydrocannabinol in adult maternally deprived rats. Neuroscience 204, 245-257.

Zamberletti E, Beggiato S, Steardo L Jr, Prini P, Antonelli T, Ferraro L, Rubino T, Parolaro D. 2014. Alterations of prefrontal cortex GABAergic transmission in the complex psychotic-like phenotype induced by adolescent delta-9-tetrahydrocannabinol exposure in rats. Neurobiol Dis 63, 35-47. 


\section{Figure legends}

\section{Figure 1}

Adolescent THC treatment does not modify fear memory processing in adult mice. (A) Schematic representation of the experimental design. Body weight of adolescent (B) male mice during the 15 days of treatment with THC (PND 35-39: $3 \mathrm{mg} / \mathrm{kg}$, PND 40-44: 6 mg/kg, and PND 45-49: 12 mg/kg) or vehicle. Locomotor activity expressed as horizontal counts of adult (C) mice after adolescent THC or vehicle treatment. (D) Anxiety-like behaviour in the EPM expressed as the percentage of time spent in the open arm of adult mice after adolescent THC or vehicle treatment. (E) Freezing levels scored during contextual fear conditioning in adult mice treated with THC or vehicle during adolescence. (F) Freezing levels scored during cued fear conditioning (S1) in adult mice treated with THC or vehicle during adolescence. (F) Time course of the freezing levels scored during cued fear extinction trials in adult mice exposed to THC or vehicle during adolescence. Data are expressed as mean \pm SEM ( $=10$ mice per group). $\star p<0.05$ (comparison between THC and vehicle). PND: postnatal day. EPM: elevated plus maze. S: Session.

\section{Figure 2}

Concomitant THC and stress exposure during adolescence impairs fear extinction in adulthood. (A) Schematic representation of the experimental design for (B-G). (B) Body weight of adolescent male mice during the 15 days of treatment with THC (PND 35-39: 3 mg/kg, PND 40-44: 6 mg/kg, and PND 45-49: 12 mg/kg) and stress exposure (n = 1516 mice per group). (C) Locomotor activity expressed as horizontal counts and (D) 
anxiety-like behaviour in the EPM expressed as the percentage of time spent in the open arm in adult mice after adolescent THC, stress, or concomitant THC/stress exposure ( $\mathrm{n}=$ 8 mice per group). (E,F) Freezing levels scored during (E) contextual and (F) cued fear conditioning (S1) in adult mice after adolescent THC, stress, or concomitant THC/stress exposure ( $n=15$-16 mice per group). (F) Time course of the freezing levels scored during cued fear extinction trials in adult mice after adolescent THC, stress, or concomitant THC/stress exposure ( $n=15-16$ mice per group). (G) AUC values for the percentage of freezing during cued fear extinction trials. Data are expressed as mean $\pm \mathrm{SEM} . \star p<0.05$; $\star \star p<0.01$ (comparison between THC and vehicle groups in (B); comparison between stress and non-stress mice in (D); comparison between THC/stress and THC/non-stress mice in (F) and (G)). PND: postnatal day. EPM: elevated plus maze. S: session. (Fisher LSD test).

\section{Figure 3}

Concomitant THC and stress exposure during adolescence increases corticosterone plasma levels after cued fear conditioning in adulthood. (A) Schematic representation of the experimental design for (B-E). Blood samples were collected at different time points, as indicated by the asterisk, and plasma corticosterone levels were measured (B) immediately after restraint (PND 49), 30 min after (C) fear conditioning training (PND 72), (D) cued fear conditioning (session 1) (PND 74) and (E) cued fear extinction (session 5) (PND 78). Data are expressed as mean \pm SEM ( $n=7-8$ mice per group). $\star \star p<0.01$ (comparison between stress and non-stress mice in (B); comparison between THC/stress and THC/non-stress mice in (D)). PND: postnatal day. (Fisher LSD test). 


\section{Figure 4}

Impaired fear extinction in adulthood induced by concomitant adolescent THC and stress exposure is associated with a reduced activity of infralimbic prefrontal cortex and basolateral amygdala. (A,C) Density of cFos-expressing cells in (A) IL and (C) BLA 2 h after the last cue extinction session of adult mice exposed to THC, stress, or concomitant THC/stress during adolescence ( $\mathrm{n}=8-9$ mice per group). (B,D) Schematic representation of the anatomical location of (B) IL and (D) BLA adapted from Paxinos and Franklin's stereotaxic atlas (Paxinos and Franklin, 2001), and representative images of both regions obtained by fluorescence microscopy after direct labelling with rabbit polyclonal antiserum to cFos. (E,F) Correlation between fear memory (freezing values) and (E) IL and (F) BLA activity (density of cFos+ cells) after the last cue extinction session. Data are expressed as mean \pm SEM. $\star p<0.05$; $\star \star p<0.01$ (comparison between THC/stress and THC/non stress mice). IL: infralimbic prefrontal cortex. BLA: basolateral amygdala. (Fisher LSD test).

\section{Figure 5}

Impaired fear extinction in adulthood induced by concomitant adolescent THC and stress exposure is associated with structural plasticity alterations in the basolateral amygdala. (A) Overall dendritic spine density, (B) analysis of spine morphology and (C) representative DiOlistics staining of BLA pyramidal neurons after the last cue extinction session of adult mice exposed to THC, stress, or concomitant THC/stress during adolescence ( $n=6-8$ mice per group). Arrows indicate thin (inmature) spines. Scale bar represents $2 \mu \mathrm{m}$. Data are expressed as mean \pm SEM. $\star p<0.05$ (comparison between 
THC/stress and THC/non stress mice); $\star \star p<0.01$ (comparison between THC and vehicle groups). BLA: basolateral amygdala. (Fisher LSD test). 